\title{
Evolução da qualidade das informações das declarações de óbito com menções de sífilis congênita nos óbitos perinatais no Brasil
}

\author{
Evolution of quality of information in death certificates \\ mentioning congenital syphilis in perinatal deaths in Brazil
}

\author{
Andréa Casagrande Azevedo ${ }^{1}$, Eliane de Freitas Drumond², \\ Rafael Valério Gonçalves ${ }^{3}$, Carla Jorge Machado ${ }^{4}$
}

\begin{abstract}
Resumo
Objetivo: avaliar a qualidade das informações das declarações de óbito (DO) com menções de sífilis congênita (SC) nos óbitos perinatais no Brasil (2001/2002 e 2012/2013). Métodos: estudo transversal com dados do Sistema de Informações de Mortalidade de óbitos fetais e neonatais precoces. Foram calculadas taxas de mortalidade fetal, neonatal precoce e perinatal específicas por SC, percentual de incompletude por variáveis selecionadas, razões de causa básica e causa múltipla (CB/CM) e outros indicadores. Analisou-se causa básica original comparativamente à causa básica após investigação. Resultados: em 2001/02 e 2012/13, ocorreram 330 e 933 óbitos perinatais, respectivamente, com menção de SC; a taxa de mortalidade perinatal passou de 4,2 a 12,8 por 100 mil nascimentos totais. A completude dos principais campos de preenchimento obrigatório melhorou, com $\mathrm{CB} / \mathrm{CM}$ de 0,8 para SC, indicando registro adequado dessa causa com as causas múltiplas. A participação da SC como causa básica da morte aumentou pós-investigação, passando de $31,2 \%$ para $85,4 \%$ (óbitos fetais) e de $54,5 \%$ a $76,2 \%$ (neonatais precoces). Conclusão: a taxa de mortalidade perinatal por SC aumentou, com melhora do preenchimento das DO. O uso das causas múltiplas de morte permitiu análise dos óbitos perinatais com menção de SC, o que é importante, dados os esforços na redução dessa doença.

Palavras-chave: sífilis congênita; declaração de óbito; causas de óbito; morte fetal; mortalidade infantil.
\end{abstract}

\begin{abstract}
Objective: Evaluate the quality of information in death certificates (DC) mentioning congenital syphilis (CS) in perinatal deaths in Brazil (2001/02 and 2012/13). Methods: Crossectional study with data from the Mortality Information System about fetal and early neonates. Fetal, early neonatal and perinatal mortality rates specific by CS, percentage of incompleteness by selected variables, underlying causes and multiple causes of death ratio $(\mathrm{CB} / \mathrm{CM})$, and other indicators were calculated. The original underlying cause was compared to the underlying cause after investigation. Results: In 2001/02 and 2012/13, 330 and 933 perinatal deaths, respectively, with CS occurred; perinatal mortality rate increased from 4.2 to 12.8 per 100.000 total births. The completion of the main required fields has improved. The $\mathrm{CB} / \mathrm{CM}$ ratio was 0.8 for $\mathrm{CS}$, indicating adequate registration of this cause, with use of multiple causes. CS percentage as the underlying cause increased post-investigation, from $31.2 \%$ to $85.4 \%$ (fetal deaths) and
\end{abstract}

${ }^{1}$ Secretaria Municipal de Saúde de Nova Lima - Nova Lima (MG), Brasil.

${ }^{2}$ Secretaria Municipal de Saúde de Belo Horizonte - Belo Horizonte (MG), Brasil.

${ }^{3}$ Faculdade de Medicina, Universidade Federal de Minas Gerais (UFMG) - Belo Horizonte (MG), Brasil.

${ }^{4}$ Departamento de Medicina Preventiva e Social, Faculdade de Medicina, Universidade Federal de Minas Gerais (UFMG) - Belo Horizonte (MG), Brasil.

Trabalho realizado na Faculdade de Medicina (FM), Universidade Federal de Minas Gerais (UFMG) - Belo Horizonte (MG), Brasil.

Endereço para correspondência: Carla Jorge Machado - Departamento de Medicina Preventiva e Social, Universidade Federal de Minas Gerais (UFMG),

Avenida Alfredo Balena, 190, Sala 701 - Santa Efigênia - CEP: 30130-100 - Belo Horizonte (MG), Brasil - Email: carlajmachado@gmail.com

Fonte de financiamento: CNPq (307237-2013-3 e 302899/2016-2); Dados do Sistema de Informação sobre Mortalidade do DATASUS.

Conflito de interesses: nada a declarar 
from $54.5 \%$ to $76.2 \%$ (early neonates). Conclusion: perinatal mortality rate due to CS increased over the study period. There was improvement in the completion of the DC. The use of multiple causes of death allowed the analysis of all perinatal deaths with CS mention, which is important given efforts in reducing CS.

Keywords: congenital syphilis; death certificates; causes of death; fetal death; infant mortality.

\section{INTRODUÇÃO}

A sífilis é uma doença importante na Saúde Pública, pois pode ser transmitida por via sexual, placentária (vertical) ou transfusão sanguínea. A sífilis congênita (SC) ocorre quando a gestante, não tratada ou inadequadamente tratada, transmite a sífilis (Treponema pallidum) para o concepto por via transplacentária, podendo trazer consequências graves à gravidez em até $80 \%$ dos casos. É uma das doenças com maiores taxas de transmissão vertical, de modo que, no Brasil, é de $34,3 \%^{1-3}$.

Calcula-se que, anualmente, dois milhões de gestantes são infectadas no mundo e cerca de 50\% das mulheres não tratadas podem transmitir a doença ao feto, com desfechos como óbitos fetal e neonatal, prematuridade, baixo peso ao nascer ou infecção congênita ${ }^{4}$. A mortalidade por SC pode atingir até $40 \%$ das crianças infectadas ${ }^{2}$. A mortalidade perinatal (óbitos fetais e neonatais precoce) é um dos indicadores mais recomendados para avaliação das assistências obstétrica e neonatal de serviços de saúde ${ }^{5}$.

Estratégias e ações visando à redução da transmissão mãefilho de HIV e sífilis foram intensificadas na década de 2000 , com a instituição da obrigatoriedade de notificação de casos ao Sistema Nacional de Agravos de Notificação (Sinan) e a celebração do Pacto pela Vida, que tem entre suas prioridades a redução das mortalidades materna e infantil ${ }^{6}$. Ressalta-se também a assinatura na Organização Pan-Americana da Saúde/Organização Mundial da Saúde (Opas/OMS) de um acordo para eliminar essa transmissão nas Américas? .

Para a eliminação da SC como problema de saúde pública, a OMS estipulou taxa de incidência a ser alcançada de 0,5 caso (incluindo natimortos) por mil nascidos vivos até $2015^{8}$. Entre os principais objetivos programáticos do acordo, está a ampliação da cobertura do pré-natal, da detecção e do tratamento da doença, com tratamento imediato, conforme protocolo, de gestantes e seus parceiros com diagnóstico de sífilis ${ }^{8,9}$. Assim, a SC é considerada indicador sentinela na qualidade da assistência ao pré-natal ${ }^{10}$. O início tardio do pré-natal e o tratamento inadequado das gestantes diagnosticadas com sífilis são fatores associados a altas taxas de $\mathrm{SC}^{11,12}$.

Em 2015, o Brasil teve taxa de incidência de SC de 6,5 casos por mil nascidos vivos em comparação com 2006, em que a taxa foi de 2 casos por mil nascidos vivos. Aumento semelhante ocorreu na taxa de mortalidade por SC. Em 2006, o País apresentou taxa de 2,3 óbitos por 100 mil nascidos vivos e passou para 7,4 óbitos em $2015^{13}$. O incremento mencionado foi relatado também nos Estados Unidos, onde a taxa passou de 8,4 casos por 100 mil nascidos vivos (2008/12) para 11,6 casos em $2014^{14}$.

Apesar da melhora na disponibilidade e qualidade das informações do Sinan, grande número de casos não é notificado, com subnotificação que varia de $25 \%$ a $64 \%^{11,15}$. Quanto à subnotificação no Sistema de Informações sobre Mortalidade (SIM) especificamente para SC, um estudo mostrou que dos 20 óbitos encontrados no Sinan, apenas dois foram registrados no SIM ${ }^{16}$, o qual constitui uma fonte alternativa de dados, fornecendo causas básica, intermediária e imediata da morte, que devem estar ligadas em uma sequência de causa e efeito. Investigações de óbitos, especialmente os maternos e infantis, são preconizadas e incentivadas pelo Ministério da Saúde (MS) e um dos objetivos é estabelecer a melhor sequência de eventos mórbidos que levou à morte, para esclarecer qual a participação de fatores modificáveis que contribuíram para a sua ocorrência ${ }^{5}$.

De acordo com a Classificação Estatística Internacional de Doenças e Problemas Relacionados à Saúde, décima revisão (CID-10), todas as doenças, afecções mórbidas ou lesões que produziram a morte devem ser registradas, com o cuidadoso estabelecimento da causa básica após a análise de cada caso ${ }^{17}$.

Este estudo tem o objetivo de avaliar a evolução da qualidade das informações das declarações de óbito com menções de SC, como causa básica ou associada (causas múltiplas), nos óbitos perinatais no Brasil em 2001/2002 e 2012/2013.

\section{MÉTODOS}

Trata-se de um estudo transversal que utilizou dados secundários do SIM. Os arquivos selecionados foram de óbitos ocorridos no Brasil em 2001, 2002, 2012, 2013 e, em seguida, conformaram-se dois biênios com os anos consecutivos. Os arquivos disponibilizados são do tipo DBC, baixados e descompactados com software Tabwin. Os arquivos foram convertidos em DBF e os dados, manipulados e analisados por planilha eletrônica Microsoft Office Excel 2010 e software Stata/SE 12.0 for Mac (64-bit Intel).

Os critérios de inclusão foram óbitos perinatais (fetais com peso igual ou superior a 500 gramas e neonatais até seis dias completos de vida), com menção dos códigos da CID-10 contidos no intervalo de A50.0 a A50.9, em qualquer linha da declaração de óbito. Já os critérios de exclusão foram óbitos fetais com peso ignorado e óbitos sem descrição do município e/ou estado de residência. Todas as análises foram estratificadas por biênio e tipo de óbito (fetal ou neonatal precoce). 
Foram analisadas (valores absolutos e percentuais) as variáveis sociodemográficas (sexo, idade e escolaridade da mãe) e da gestação, do parto e do feto/recém-nascido (filhos tidos nascidos vivos e filhos tidos nascidos mortos, gravidez, gestação em semanas, via de parto, peso ao nascer em gramas). As variáveis relativas ao óbito foram óbito no parto, assistência médica, necropsia, atestante médico, óbito investigado e local de ocorrência. Essas variáveis foram categorizadas em com informação e sem informação (ou dados incompletos). A categoria "dados incompletos" incluiu qualquer dado faltante (missing/casela vazia/dado em branco) ou categoria específica para dado não especificado/não declarado/ignorado (como 9 ou 99). Assim, o percentual de incompletude de cada variável foi calculado sendo categorizado conforme estudo prévio $^{18}$ : excelente (inferior a $5 \%$ de preenchimento incompleto), bom (5\% a 10\%), regular (10\% a menos de $20 \%$ ), ruim (20\% a menos de $50 \%)$ e muito ruim (50\% ou mais).

Considerou-se ser uma causa distinta das demais quando cada um dos quatro caracteres fosse distinto dos outros, ou seja, foram consideradas as causas definidas por classificação em nível de quatro caracteres da lista tabular da CID-10. Em seguida, foram contadas todas as causas básicas $(\mathrm{CB})$ e as causas múltiplas (básicas mais associadas: CM) mencionadas nos óbitos fetais e neonatais precoces nas declarações por período e apenas uma causa foi contada em determinada categoria a quatro caracteres se fossem informadas duas ou mais vezes no mesmo atestado de óbito. Assim, a duplicação ou repetição de causas foi eliminada.

Ainda que improvável, tal duplicação/repetição em grupamentos tão específicos (quatro caracteres), esse rigor foi necessário após a verificação inicial de menção de P95.X ou A50.9 (morte fetal de causa não especificada ou SC não especificada) duas vezes na mesma declaração, o que suscitou suspeita de que isso ocorresse outras vezes. Após esse procedimento, para apresentação das causas, foram feitos grupos, a partir da ClD-10: P00.0 a P04.9 (feto e recém-nascido afetados por fatores maternos e por complicações da gravidez, do trabalho de parto e do parto); P05.0 a P08.2 (transtornos relacionados com a duração da gestação e o crescimento fetal); P20.0 a P29.9 (transtornos respiratórios e cardiovasculares específicos do período perinatal); P35.0 a P39.9 (infecções específicas do período perinatal); P50.0 a P61.9 (transtornos hemorrágicos e hematológicos do feto e do recém-nascido); P70.0 a P74.9 (transtornos endócrinos e metabólicos transitórios específicos do feto e do recém-nascido); P75 a P78.9 (transtornos do aparelho digestivo do feto ou do recém-nascido); P80.0 a P83.9 (afecções comprometendo o tegumento e a regulação térmica do feto e do recém-nascido); P90 a P96.9 (outros transtornos originados no período perinatal); Q00.0 Q99.9 (malformações congênitas, deformidades e anomalias cromossômicas); outras (todas as demais causas não classificadas nos grupos anteriormente formados). Enquanto a contagem de $\mathrm{CB}$ totaliza o número de óbitos do estudo, a contagem de CM totaliza menções.

Após a análise da distribuição dos óbitos segundo as $\mathrm{CB}$ e do número de menções de causas, foram calculadas razões CB/CM para óbitos fetais e neonatais precoces e para cada biênio. Essa razão varia de 0 a 1 e, quanto mais próxima da unidade, mais uma causa é mencionada como básica. Foi realizado, ainda, o cálculo do número médio de menções por DO, dividindo-se a soma de todas as menções pelo número de óbitos.

Finalmente, analisou-se a causa básica original comparativamente à básica após a investigação do óbito. Foi realizada distribuição das CB mencionadas em cada campo da DO. Essa análise foi realizada apenas para 2012/2013, em que os campos pré e pós-investigação foram disponibilizados pela base de dados.

Nesse estudo, também foram calculadas taxas de mortalidade específicas por SC nos componentes fetal, neonatal precoce e perinatal. Para óbitos neonatais precoces, dividiu-se o número de óbitos neonatais precoces pelo número de nascidos vivos. Para óbitos fetais, a taxa foi calculada dividindo-se o número de óbitos fetais pela soma do número de nascidos vivos e óbitos fetais (estimativa de nascimentos totais de mães residentes). Para óbitos perinatais, o denominador da taxa consistiu na soma de óbitos fetais e neonatais precoces dividido pela soma do número de nascidos vivos e óbitos fetais (estimativa de nascimentos totais de mães residentes) conforme o Manual de vigilância do óbito infantil e fetal e do Comitê de Prevenção do Óbito Infantil e Fetal ${ }^{2}$. Posteriormente, multiplicou-se a taxa por 100 mil. Para manter a comparabilidade das taxas calculadas com a literatura, foram utilizados apenas óbitos cujas causas básicas estavam no intervalo A50.0 a A50.9.

A pesquisa foi submetida ao Comitê de Ética e Pesquisa da Universidade Federal de Minas Gerais em 10 de março de 2016, sendo aprovada de acordo com CAAE: 54067716.1.0000.5149.

\section{RESULTADOS}

Em relação aos óbitos fetais por SC (códigos A50.0 a A50.9), essa causa foi considerada básica em 153 e 543 óbitos fetais em 2001/2002 e 2012/2013, respectivamente. No caso dos neonatais precoces, a SC foi a causa básica em 111 e 210 dos óbitos neonatais precoces, respectivamente, em 2001/2002 e 2012/2013. Assim, ocorreram 1.017 óbitos perinatais em que a SC foi causa básica. Consideradas 1.295 menções de SC, recuperou-se $27 \%$ do total inicial de óbitos perinatais $(1.295-1.017=278)$.

As taxas de mortalidade específicas por SC foram, no período fetal, em 2001/2002 e 2012/2013, respectivamente, 2,45 e 9,24 óbitos por 100 mil nascidos totais; no período neonatal precoce, 1,80 e 3,61 óbitos por 100 mil nascidos vivos. A taxa de mortalidade perinatal foi, em 2001/2002 e 2012/2013, 4,22 e 12,82 por 100 mil nascimentos totais, respectivamente. 
A Tabela 1 descreve o percentual de incompletude de preenchimento dos óbitos perinatais (fetais e neonatais precoce) em 2001/2002 e 2012/2013. Aumentou a participação dos óbitos fetais em $22 \%$ e decresceu a dos óbitos neonatais precoce em 28,4\% no biênio de 2012/2013.

Conforme os escores de classificação da incompletude ${ }^{18}$, houve melhora da idade da mãe, número de filhos tidos nascidos vivos, número de filhos tidos nascidos mortos e peso ao nascer, o tipo de parto, óbito em relação ao parto e atestante do óbito (Tabela 1). Houve piora entre 2001/2002 e 2012/2013 para idade gestacional (óbitos neonatais precoces) e necropsia (óbitos fetais) (Tabela 1). Não houve mudança na classificação para sexo, tipo de gravidez e local de ocorrência (óbitos fetais e neonatais precoces), tipo de parto (óbitos fetais), idade gestacional (neonatais precoces), assistência médica (óbitos fetais e neonatais precoces) e necropsia (óbitos neonatais precoces). Além do já assinalado na Tabela 1, ressalta-se a proporção de óbitos fetais com sexo classificado como ignorado, que foi $11,8 \%$ em $2012 / 2013$, comparado a $1,6 \%$ em $2001 / 2002$, ou seja, aumento de mais de seis vezes no período.

Na Tabela 2, estão apresentados os óbitos fetais com menção de SC. O valor total $(\mathrm{n}=186)$ expresso na última linha da coluna causa básica (CB) são óbitos com menção de SC (A50.0 a A50.9) em qualquer linha da DO. Assim, enquanto em 2001/2002 havia 153 óbitos cuja causa básica de óbito foi a SC, houve adicionais 33 óbitos nos quais a causa básica não foi SC: 14 óbitos cuja causa básica foi do grupo P00.0 a P04.9 (feto e recém-nascido afetados por fatores maternos e por complicações da gravidez, do trabalho de parto e do parto); 14 cuja causa básica foi
P20.0 a P29.9 (transtornos respiratórios e cardiovasculares específicos do período perinatal).

Na coluna CM (causas múltiplas), está representado o número de vezes que as causas foram mencionadas na DO ( $\mathrm{n}=347)$. Assim, ocorreram 189 menções de SC, 35 menções de P00.0 a P04.9, 104 menções de P20.0 a P29.9 e, assim, sucessivamente, ou seja, houve 347 menções de diferentes causas, implicando média de 1,87 menção de causas por DO $(347 / 186=1,87)$. Finalmente, as razões CB/CM indicam a importância de causa como causa básica ou causa intermediária ou imediata de morte. Exemplo de como aferir essa importância se refere às causas do grupo P20.0 a P29.9, consideradas causas básicas em 14 óbitos, mas mencionadas 104 vezes em qualquer campo da DO, ou seja, razão CB/CM foi igual a 0,1 , indicando que foi causa básica em apenas $10 \%$ das vezes que foi mencionada. Os dados de óbitos fetais para 2012/2013 e para óbitos neonatais precoces nos dois biênios podem ser analisados de forma similar (Tabela 2).

Desses dados, observa-se que, para óbitos fetais, entre aqueles com menção de SC, a participação da sífilis como causa básica foi elevada e teve leve acréscimo, passando de $82,3 \%$ em $2001 / 2002(153 / 186=0,823)$ a $84,7 \%$ em $2012 / 2013(543 / 642=0,847)$; a segunda causa básica mais frequente entre óbitos com menção de sífilis foi P00.0 a P04.9; $7,5 \%(n=14)$ e $10,4 \%(n=67)$ em 2001/2002 e 2012/2013, respectivamente. Em 2012/2013, as malformações congênitas (Q00.0 a Q99.9) foram a terceira causa básica de morte fetal mais frequente $(1,9 \% ; \mathrm{n}=12)$, seguidas do grupo P20.0 a P20.9 ( $\mathrm{n}=11 ; 1,7 \%)$ (Tabela 2).

Tabela 1. Percentual de incompletude de preenchimento das características sociodemográficas, maternas e relacionadas ao óbito: óbitos fetais e neonatais precoces com menção de sífilis congênita na Declaração de Óbito. Brasil, biênios 2001/2002 e 2012/2013

\section{Variáveis}

\section{Sociodemográficas}

Sexo

Faixa etária da mãe (em anos)

Escolaridade da mãe (em anos)

Da gestação/parto

Número de filhos vivos

Quantidade de filhos mortos

Gravidez

Gestação (em semanas)

Peso ao nascer (em gramas)

Parto

Do óbito

Local de ocorrência

Óbito em relação ao parto

Assistência médica

Necropsia

Atestante

2001/02

Fetal $(\mathrm{N}=186) \quad \begin{gathered}\text { Neonatal precoce } \\ (\mathrm{N}=144)\end{gathered}$

$$
\text { Fetal }(N=186)
$$

0,0

9,1

28,4

35,0

29,6

2,6

7,0

10,7

3,2

0,5

5,3

66,6

42,4

11,3

0,0
16,0
35,4

31,3
45,1
0,7
8,3
9,7
7,7

1,4
16,7
49,3
36,1
5,4

0,0

6,0

5,4

31,3

45,1

0,7

8,3

9,7

, 7

1,4

16,7

49,3

6,1

5,4
2012/13

Fetal $(\mathrm{N}=642) \quad$ Neonatal precoce

$(\mathrm{N}=291)$

0,0
6,5
16,5

6,8
13,1
3,0
10,6
3,4
4,5

0,2
3,8
24,4
25,8
7,5

Fonte: Sistema de Informação sobre Mortalidade 
Quanto às menções para óbitos fetais, destaca-se que, embora tenha havido queda percentual das menções do grupo A50.0 a A50.9, que passaram de 54,5\% (189/347 = 0,545) para $48,3 \%(644 / 1.335=0,482)$, a participação da SC como causa básica cresceu de 81,0\% (123/189) para 84,3\% (644/543). Além disso, houve queda do grupo P20.0 a P20.9, cujas causas passaram de $30,0 \%$ para 24,6\%, e aumento da participação das menções de P00.0 a P04.9, que passou de $10,1 \%$ a $18,2 \%$ (Tabela 2).

Quanto às razões $\mathrm{CB} / \mathrm{CM}$ para as causas que permitiram o cálculo dessa razão (óbitos fetais), ficaram em 0,8 (A50.0-A50.9) para SC nos dois períodos. Houve redução para todas as demais causas, exceto o grupo Q00.0 a Q99.9, que passou de 0,2 a 0,5. Houve ainda mais diversidade de causas em 2012/2013, pois maior número de razões $\mathrm{CB} / \mathrm{CM}$ pôde ser calculada comparativamente a 2001/2002 (Tabela 2).

Finalmente, houve aumento do número médio de diagnósticos informados por DO no período, para óbitos fetais, passando de 1,87 para 2,08 (Tabela 2). Para óbitos neonatais precoces, pontua-se que, a despeito da melhora da qualidade da informação observada nas DO e aumento do percentual de menções da SC, que passou de $31,9 \%(141 / 442=0,319)$ para $38,1 \%(321 / 842=0,381)$ do total de menções, houve queda da participação da sua seleção como causa básica, de 77,1\% (111/144 = 0,771) para 72,2\% $(210 / 291=0,722)$. Chama atenção o aumento da seleção, como causa básica, de doenças do grupo P00.0 a P04.9 (n = 6; 4,2\% em 2001/2002 e $n=23 ; 7,9 \%$ em 2012/2013 - do qual fazem parte os afetados por doenças infecciosas e parasitárias da mãe; excluída a SC, entre outras) e manutenção do grupo P20.0 a P29.9 $(\mathrm{n}=11 ; 7,6 \%$ e $\mathrm{n}=7,6 \%)$ (Tabela 2$)$.

Quanto às menções de causas em óbitos neonatais precoces no período analisado, pode-se destacar o aumento da participação das menções de P00.0 a P04.9, que passaram de 3,4\% para 11,4\%. Chama atenção também que as causas do grupo P05.0 a P08.9 (transtornos relacionados com a duração da gestação e o crescimento fetal) não foram mencionadas em 2012/2013, mas houve 85 menções dessas causas em qualquer posição da DO em 2001/2002, perfazendo participação de 19,2\% em 2001/2002, caindo a zero em 2012/2013 (Tabela 2).

Tratando-se das razões CB/CM, passaram de 0,8 a 0,7 para SC no período, ou seja, apesar de ter sido mencionada, a sífilis não foi selecionada como causa de morte. Uma das possíveis explicações para esse fato foi que, para o grupo malformações congênitas, deformidades e anomalias cromossômicas (Q00.0 a Q99.9), a relação $\mathrm{CB} / \mathrm{CM}$ passou de 0,2 para 0,4. Também o grupo transtornos hemorrágicos e hematológicos do feto e do recém-nascido (P50.0 a P61.9) teve aumento na relação $\mathrm{CB} / \mathrm{CM}$, de 0,1 para 0,3 , respectivamente (Tabela 2 ).

Constata-se queda do número médio de menções entre 2001/2002 e 2012/2013 para óbitos neonatais precoces, passando de 3,07 para 2,89 (Tabela 2). O aumento do número de menções reflete a valorização de complicações e comorbidades na evolução para a morte.

No que se refere à investigação em 2012/2013, dos 642 óbitos fetais com menção de SC, 513 (79,9\%) foram investigados, e

Tabela 2. Número de óbitos com menção de sífilis congênita, segundo causas básicas (CB) e causas múltiplas de morte (CM) e razões CB/CM. Brasil 2001/2002 e 2012/2013

\begin{tabular}{|c|c|c|c|c|c|c|c|c|c|c|c|c|}
\hline \multirow[t]{2}{*}{ Grupo de causas } & \multicolumn{3}{|c|}{ Fetal 2001/2002 } & \multicolumn{3}{|c|}{ Fetal 2012/2013 } & \multicolumn{3}{|c|}{$\begin{array}{c}\text { Neonatal precoce } \\
2001 / 2002\end{array}$} & \multicolumn{3}{|c|}{$\begin{array}{c}\text { Neonatal precoce } \\
2012 / 2013 \\
\end{array}$} \\
\hline & CB & CM & $\mathrm{CB} / \mathrm{CM}$ & CB & $\mathbf{C M}$ & $\mathrm{CB} / \mathrm{CM}$ & CB & $\mathrm{CM}$ & $\mathrm{CB} / \mathrm{CM}$ & CB & CM & $\mathrm{CB} / \mathrm{CM}$ \\
\hline A50.0-A50.9 & 153 & 189 & 0,8 & 543 & 644 & 0,8 & 111 & 141 & 0,8 & 210 & 321 & 0,7 \\
\hline P00.0-P04.9 & 14 & 35 & 0,4 & 67 & 244 & 0,3 & 6 & 15 & 0,4 & 23 & 96 & 0,3 \\
\hline P05.0-P08.2 & 0 & 0 & $* *$ & 1 & 20 & 0,1 & 1 & 85 & 0,01 & 0 & 0 & $* *$ \\
\hline P20.0-P29.9 & 14 & 104 & 0,1 & 11 & 328 & 0,03 & 11 & 121 & 0,1 & 22 & 207 & 0,1 \\
\hline P35.0-P39.9 & 2 & 9 & 0,2 & 1 & 7 & 0,1 & 9 & 52 & 0,2 & 7 & 114 & 0,1 \\
\hline P50.0-P61.9 & 0 & 0 & $* *$ & 2 & 3 & 0,7 & 2 & 17 & 0,1 & 9 & 30 & 0,3 \\
\hline P70.0-P74.9 & 1 & 1 & 1,0 & 1 & 4 & 0,3 & 0 & 0 & $* *$ & 2 & 6 & 0,3 \\
\hline P75.0-P78.9 & 0 & 0 & $* *$ & 0 & 0 & $* *$ & 0 & 0 & $* *$ & 1 & 2 & 0,1 \\
\hline P80.0-P83.9 & 1 & 4 & 0,3 & 2 & 22 & 0,1 & 0 & 0 & $* *$ & 0 & 0 & $* *$ \\
\hline P90.0-P96.9 & 0 & 0 & $* *$ & 2 & 39 & 0,1 & 0 & 0 & $* *$ & 2 & 30 & 0,1 \\
\hline Q00.0-Q99.9 & 1 & 5 & 0,2 & 12 & 24 & 0,5 & 2 & 9 & 0,2 & 14 & 35 & 0,4 \\
\hline Outras causas & 0 & $* *$ & $* *$ & 0 & 0 & $* *$ & 2 & 2 & 1,0 & 1 & 1 & 1,0 \\
\hline Total & 186 & 347 & & 642 & 1335 & & 144 & 442 & & 291 & 842 & \\
\hline
\end{tabular}

Fonte: Sistema de Informação sobe Mortalidade. Notas: P00.0-P04.9 (feto e recém-nascido afetados por fatores maternos e complicações da gravidez, do trabalho de parto e do parto); P05.0-P08.2 (transtornos relacionados com a duração da gestação e o crescimento fetal); P20.0-P29.9 (transtornos respiratórios e cardiovasculares específicos do período perinatal); P35.0-P39.9 (infecções específicas do período perinatal); P50.0-P61.9 (transtornos hemorrágicos e hematológicos do feto e do recém-nascido); P70.0-P74.9 (transtornos endócrinos e metabólicos transitórios específicos do feto e do recém-nascido; P75-P78.9 (transtornos do aparelho digestivo do feto ou do recém-nascido); P80.0-P83.9 (afecções comprometendo o tegumento e a regulação térmica do feto e do recém-nascido); P90-P96.9 (outros transtornos originados no período perinatal); Q00.0-Q99.9 (malformações congênitas, deformidades e anomalias cromossômicas); outras (todas as demais causas não classificadas nos grupos anteriormente formados); ${ }^{* *}$ : Não calculado 
Tabela 3. Distribuição da causa básica original e após investigação de óbitos fetais com menção de sífilis congênita, segundo CID-10 17 . Brasil 2012/2013

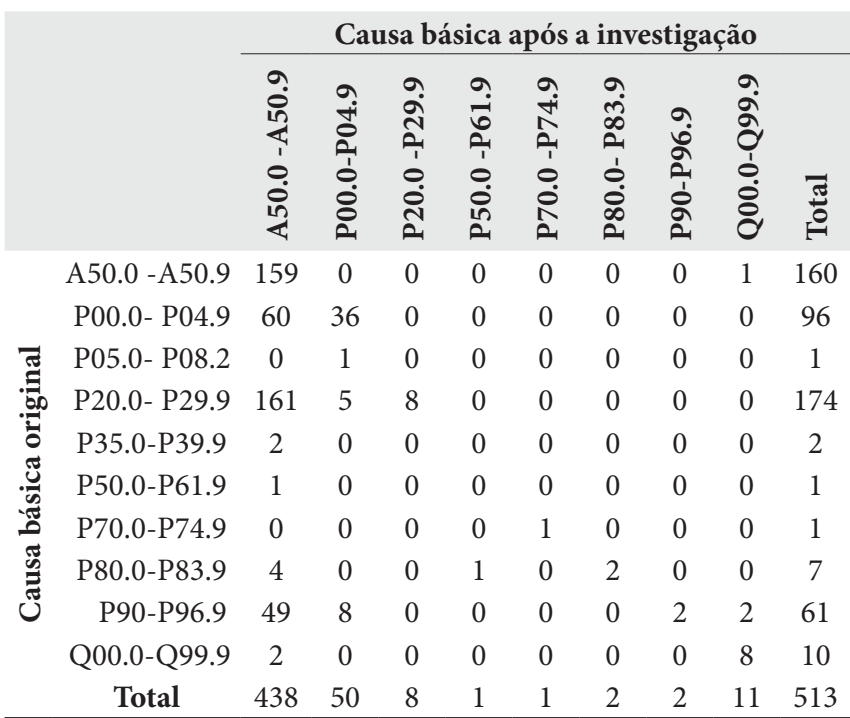

Fonte: Sistema de Informação sobre Mortalidade. Notas: P00.0 a P04.9 (feto e recém-nascido afetados por fatores maternos e por complicações da gravidez, do trabalho de parto e do parto); P05.0 a P08.2 (transtornos relacionados com a duração da gestação e com o crescimento fetal); P20.0 a P29.9 (transtornos respiratórios e cardiovasculares específicos do período perinatal); P35.0 a P39.9 (infecções específicas do período perinatal); P50.0 a P61.9 (transtornos hemorrágicos e hematológicos do feto e do recém-nascido); P70.0 a P74.9 (transtornos endócrinos e metabólicos transitórios específicos do feto e do recém-nascido; P75 a P78.9 (transtornos do aparelho digestivo do feto ou do recém-nascido); P80.0 a P83.9 (afecções comprometendo o tegumento e a regulação térmica do feto e do recém-nascido); P90 a P96.9 (outros transtornos originados no período perinatal); Q00.0 a Q99.9 (malformações congênitas, deformidades e anomalias cromossômicas)

dos 291 óbitos neonatais precoces, 189 foram investigados $(64,9 \%)$ (Tabelas 3 e 4$)$.

Antes da investigação, havia 160 óbitos fetais cuja causa básica era SC (A50.0 a A50.9) e, após a investigação, tal número passou a 438 , ou seja, houve um aumento de $173 \%$. Assim, passaram a ter como causa básica a SC após a investigação: 161 óbitos, cuja causa básica original era P20.0 a P29.9, 60 óbitos, cuja causa básica original era P00.0 a P04.9, e 49 óbitos, cuja causa básica original era P90.0 a P096.9 (feto e recém-nascido afetados por fatores maternos e complicações da gravidez, do trabalho de parto e do parto) (Tabela 3). No que se refere aos óbitos neonatais precoces, havia 103 óbitos cuja causa básica era SC antes da investigação, tendo esse número aumentado em 32,7\%, passando para 144 após a investigação. Dez óbitos, cuja causa básica original era P00.0 a P04.9, e dez óbitos, cuja causa básica original era P20.0 a P29.9, passaram a ter como causa básica a SC após a investigação.

A Tabela 5 resume o impacto das investigações na seleção das causas básicas. Destaca-se o percentual de casos em que a investigação qualificou a causa básica da morte da forma etiologicamente mais específica quanto possível. A SC (A50.0 a A50.9) aumentou em quase 50\% no caso dos
Tabela 4. Distribuição da causa básica original e após investigação de óbitos neonatais precoce com menção de sífilis congênita, segundo CID 10 ${ }^{17}$. Brasil, 2012/13

\begin{tabular}{|c|c|c|c|c|c|c|c|c|c|c|c|c|}
\hline & & \multicolumn{11}{|c|}{ Causa básica após investigação } \\
\hline & & 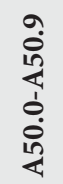 & $\stackrel{\infty}{\stackrel{\mathcal{I}}{\Xi}}$ & 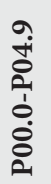 & 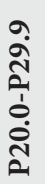 & 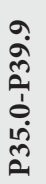 & $\begin{array}{l}9 \\
\text { - } \\
0 \\
0 \\
0 \\
0 \\
10 \\
0\end{array}$ & 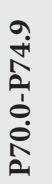 & 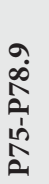 & 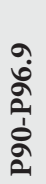 & 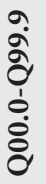 & స్ేㅇ \\
\hline \multirow{12}{*}{ 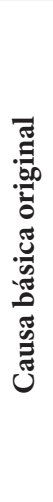 } & A50.0-A 50.9 & 101 & 0 & 0 & 0 & 0 & 1 & 0 & 0 & 0 & 1 & 103 \\
\hline & $\mathrm{I} 42.8$ & 0 & 1 & 0 & 0 & 0 & 0 & 0 & 0 & 0 & 0 & 1 \\
\hline & P00.0-P04.9 & 10 & 0 & 8 & 0 & 0 & 0 & 0 & 1 & 0 & 1 & 20 \\
\hline & P05.0-P08.2 & 7 & 0 & 2 & 0 & 0 & 0 & 0 & 0 & 0 & 0 & 9 \\
\hline & P20.0-P29.9 & 10 & 0 & 0 & 8 & 0 & 0 & 1 & 0 & 0 & 0 & 19 \\
\hline & P35.0-P39.9 & 7 & 0 & 2 & 1 & 4 & 1 & 0 & 0 & 0 & 0 & 15 \\
\hline & P50.0-P61.9 & 1 & 0 & 0 & 0 & 0 & 3 & 0 & 0 & 0 & 0 & 4 \\
\hline & P75-78.9 & 1 & 0 & 0 & 0 & 0 & 0 & 0 & 0 & 0 & 0 & 1 \\
\hline & P80.0-P83.9 & 2 & 0 & 0 & 0 & 0 & 0 & 0 & 0 & 0 & 0 & 2 \\
\hline & P90-P96.9 & 2 & 0 & 2 & 0 & 0 & 0 & 0 & 0 & 1 & 0 & 5 \\
\hline & Q00.0-Q99.9 & 3 & 0 & 0 & 0 & 0 & 0 & 0 & 0 & 0 & 7 & 10 \\
\hline & Total & 144 & 1 & 14 & 9 & 4 & 5 & 1 & 1 & 1 & 9 & 189 \\
\hline
\end{tabular}

Fonte: Sistema de Informação sobre Mortalidade. Notas: P00.0 a P04.9 (feto e recém-nascido afetados por fatores maternos e complicações da gravidez, do trabalho de parto e do parto); P05.0 a P08.2 (transtornos relacionados com a duração da gestação e o crescimento fetal); P20.0 a P29.9 (transtornos respiratórios e cardiovasculares específicos do período perinatal); P35.0 a P39.9 (infecções específicas do período perinatal); P50.0 a P61.9 (transtornos hemorrágicos e hematológicos do feto e do recém-nascido); P70.0 a P74.9 (transtornos endócrinos e metabólicos transitórios específicos do feto e do recém-nascido; P75 a P78.9 (transtornos do aparelho digestivo do feto ou do recém-nascido); P80.0 a P83.9 (afecções comprometendo o tegumento e a regulação térmica do feto e do recém-nascido); $\mathrm{P} 90$ a P96.9 (outros transtornos originados no período perinatal); Q00.0 a Q99.9 (malformações congênitas, deformidades e anomalias cromossômicas); outras (todas as demais causas não classificadas nos grupos anteriormente formados)

óbitos neonatais precoces e em mais de $150 \%$ em óbitos fetais (Tabela 5).

\section{DISCUSSÃO}

Este trabalho indicou aprimoramento da qualidade das informações sobre óbitos perinatais com menções de SC, como causa básica ou associada (causas múltiplas) no Brasil em 2001/2002 e 2012/2013. Observou-se, diferentemente do já relatado ${ }^{19,20}$, que os óbitos fetais não possuem informações mais mal preenchidas do que os neonatais precoces. A abordagem das causas múltiplas de óbitos permitiu a ampliação da importância da SC entre as causas de morte perinatal no Brasil, tendo em vista que maior número de óbitos perinatais com menção de SC foi recuperado.

A magnitude das taxas de mortalidade perinatal por SC é alarmante, o que justifica estudos como este, que avalia a qualidade das informações de óbito, o que, de fato, tem sido objeto de contínuos esforços para melhoria, a fim de garantir a fidedignidade dos indicadores no Brasil ${ }^{21}$. As taxas de mortalidades fetal e neonatal precoce por SC apresentaram aumento marcante 
Tabela 5. Distribuição proporcional das causas básicas originais e após investigação de óbitos fetais e neonatais precoce, com menção de sífilis congênita investigados, segundo CID-1017. Brasil 2012/2013

\begin{tabular}{|c|c|c|c|c|c|c|c|c|}
\hline \multirow{3}{*}{$\begin{array}{c}\text { Grupos } \\
\text { CID-10 } \\
50.0 \text { a A50.9 }\end{array}$} & \multicolumn{4}{|c|}{$\begin{array}{l}\text { Óbitos fetais } \\
(\mathrm{n}=513 ; 100 \%)\end{array}$} & \multicolumn{4}{|c|}{$\begin{array}{c}\text { Óbitos neonatais } \\
\text { precoces } \\
(\mathbf{n}=189 ; 100 \%)\end{array}$} \\
\hline & \multicolumn{2}{|c|}{$\begin{array}{c}\text { CB } \\
\text { original }\end{array}$} & \multicolumn{2}{|c|}{$\begin{array}{c}\text { CB após } \\
\text { investigação }\end{array}$} & \multicolumn{2}{|c|}{$\begin{array}{c}\mathrm{CB} \\
\text { original }\end{array}$} & \multicolumn{2}{|c|}{$\begin{array}{c}\text { CB após } \\
\text { investigação }\end{array}$} \\
\hline & 160 & 31,2 & 438 & 85,4 & 103 & 54,5 & 144 & 76,2 \\
\hline I 42.8 & 0 & ( & 0 & 0,0 & 1 & 0,5 & 1 & 0,5 \\
\hline P00.0 a P04.9 & 96 & 18,7 & 50 & 9,7 & 20 & 10,6 & 14 & 7,4 \\
\hline P05.0 a & 1 & & 0 & 0,0 & 9 & 4,8 & 0 & 0,0 \\
\hline P20.0 a P29.9 & 174 & 3 & 8 & & 19 & 10,1 & 9 & $4, \varepsilon$ \\
\hline P35.0 a P39.9 & 2 & 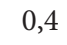 & 0 & م & 15 & 7,9 & 4 & 2 , \\
\hline P50.0 a P61.9 & 1 & 0 & 1 & 0 & $T$ & 2,1 & 5 & 2,6 \\
\hline P70.0 a P74.9 & 1 & 0 & 1 & . & 0 & 0,0 & 1 & 0,5 \\
\hline P75 a P78.9 & 0 & 0 & 0 & م & 1 & 0,5 & 1 & 0,5 \\
\hline P80.0 a P83.9 & 7 & 1,4 & 2 & 0,4 & 2 & 0,5 & 0 & 0,0 \\
\hline P90 a P96.9 & 61 & 11,9 & 2 & 0,4 & 5 & 2,6 & 1 & 0,5 \\
\hline Q00.0 a Q99.9 & 10 & 1,9 & 11 & 2,1 & 10 & 5,3 & 9 & 4 , \\
\hline
\end{tabular}

Fonte: Sistema de Informação sobre Mortalidade. Notas: P00.0 a P04.9 (feto e recém-nascido afetados por fatores maternos e complicações da gravidez, do trabalho de parto e do parto); P05.0 a P08.2 (transtornos relacionados com a duração da gestação e o crescimento fetal); P20.0 a P29.9 (transtornos respiratórios e cardiovasculares específicos do período perinatal); P35.0 a P39.9 (infecções específicas do período perinatal); P50.0 a P61.9 (transtornos hemorrágicos e hematológicos do feto e do recém-nascido); P70.0 a P74.9 (transtornos endócrinos e metabólicos transitórios específicos do feto e do recém-nascido; P75 a P78.9 (transtornos do aparelho digestivo do feto ou do recém-nascido); P80.0 a P83.9 (afecções comprometendo o tegumento e a regulação térmica do feto e do recém-nascido); P90 a P96.9 (outros transtornos originados no período perinatal); Q00.0 a Q99.9 (malformações congênitas, deformidades e anomalias cromossômicas); outras (todas as demais causas não classificadas nos grupos anteriormente formados)

no período estudado. $\mathrm{O}$ aumento da taxa de mortalidade infantil por SC foi descrito no Boletim Epidemiológico do MS, com taxas de 2,8 por 100 mil nascidos vivos em 2001 e 5,5 por 100 mil em 2013, de modo que os achados do presente estudo corroboram o aumento observado e, ainda, apontam para o fato de que a mortalidade fetal é a mais crítica, sendo 3,7 vezes, em 2012/2013, a taxa de 2001/029. De fato, estudos evidenciam a SC como importante causa de morte fetal ${ }^{22,23}$.

Em relação à incompletude, houve melhora geral do preenchimento dos dados do primeiro biênio para o segundo, nos óbitos fetais e nos neonatais precoces. Essa melhora ocorreu, exceção feita à idade gestacional no período neonatal, para todas as variáveis de preenchimento compulsório (idade da mãe, escolaridade da mãe, filhos nascidos vivos, filhos nascidos mortos, tipo de gravidez, peso ao nascer, tipo de parto e óbito em relação ao parto $)^{24}$. Essa tendência de aprimoramento na completude das informações também foi observada em outros estudos que utilizaram declaração de óbito ${ }^{20}$. Chama a atenção, contudo, que, dessas variáveis, ainda em 2012/2013, havia percentuais de incompletude para escolaridade da mãe, número de filhos tidos nascidos mortos e idade gestacional superiores a $10 \%$, havendo espaço para aprimoramento da coleta e registro dessas informações. Esse percentual é preocupante, pois, no caso de escolaridade, reflete a condição socioeconômica da mãe, sendo uma das variáveis mais utilizadas na avaliação da desigualdade social, a qual é, muitas vezes, preenchida por pessoal administrativo ${ }^{18,20}$.

O número de menções é um indicador de qualidade do preenchimento da $\mathrm{DO}$, pois quanto maior, melhor espera-se que seja a qualidade da informação contida na $\mathrm{DO}^{25}$. Embora tenha aumentado nos óbitos fetais entre os biênios, o número médio de menções de causas por DO obtido (de 2,5 menções) é inferior à média nacional de 2003 (2,8 menções), refletindo a magnitude da perda da informação sobre causas de morte ao se utilizar apenas a causa básica ${ }^{25}$. Além disso, é preocupante a queda de menções em óbitos neonatais precoces, ou seja, ainda que tenha melhorado no período, ainda há espaço para que sejam declaradas mais causas de óbito na DO.

As razões $(\mathrm{CB} / \mathrm{CM})$ mensuraram a importância de cada patologia como causa básica ou consequencial. Por meio dela, pode-se observar que tanto para os anos de 2001/2002 como para 2012/2013 a SC estaria "escondida" ou subnotificada em $70 \%$ a $80 \%$ dos óbitos que envolvem a SC. Um dos fatores para explicar essa ocorrência é que a aplicação das regras de codificação e de seleção leva, em alguns casos, à não seleção da causa básica escrita pelo médico na $\mathrm{DO}$, como causa básica da morte.

A avaliação das causas básicas antes e após a investigação do óbito foi objeto de outros estudos ${ }^{26-28}$. Aqui se percebeu um aumento da seleção da SC como causa básica após a investigação. Estudos sobre investigação das causas encontraram maior atribuição a causas maternas, inclusive a sífilis, na cadeia causal do óbito fetal ${ }^{29}$. Melhoria da qualidade da informação sobre a causa básica da morte, após a investigação, pode ser observada pela diminuição da seleção como causa básica. Em patologias que devem ser consideradas "consequências de", como as do grupo P20.0 a 29.9 (transtornos respiratórios e cardiovasculares específicos do período perinatal), por exemplo, houve queda de $93,9 \%$ na sua seleção como causa básica. Esse resultado corrobora um estudo realizado em Londrina ${ }^{27}$, no qual, das 46 causas desse grupo mencionadas originalmente na DO, apenas seis permaneceram após a investigação. Por outro lado, existe a possibilidade de erro de codificação nas menções do grupo P00.0 a P04.9 (feto e recém-nascidos afetados por fatores maternos e por complicações da gravidez do trabalho de parto e do parto), no qual não se inclui a sífilis. A maior especificidade das causas de morte obtida após investigação pode ser também observada na grande diminuição (97\%) das causas do grupo 
P90 a P96, visto que o código P95 (morte fetal de causa não especificada) está nele incluído.

Uma limitação do presente estudo é a utilização apenas do SIM como fonte de dados. Além disso, esse trabalho não abordou diferenças regionais, o que torna mais difícil a delimitação de políticas focalizadas para a prevenção da SC. Contudo, os resultados do estudo já apontam que o Brasil ainda está longe de cumprir a meta de eliminação da SC, de modo que a persistência da doença entre as gestantes, seja por problemas no acesso ao pré-natal de qualidade, problemas no diagnóstico, seja por dificuldades no tratamento tempestivo e adequado da mulher e de seu parceiro, tem resultado em elevadas taxas de mortalidades fetal e neonatal. A implantação da Rede Cegonha, a qual assegura uma rede de cuidados à gestante, a disponibilidade dos testes rápidos de sífilis e de HIV, é uma forma eficaz para a prevenção e o tratamento oportuno dessas doenças ${ }^{2}$.
Para um tratamento sem riscos de transmissão da sífilis ao feto, as gestantes e o(s) parceiro(s) sexual(is) devem ser tratados corretamente ${ }^{8}$ e o tratamento baseia-se na prescrição de penicilina $\mathrm{G}$ benzatina, realizada em até três doses, conforme estágio da doença na gestante e parceiro $(s)^{1}$. Apesar dos avanços já observados, deficiências na qualidade da informação sobre a SC, considerada evento sentinela da qualidade da assistência pré-natal, são ainda significativas no País.

\section{CONCLUSÃO}

O estudo aponta para a necessidade de orientação dos médicos para o adequado preenchimento das declarações de óbitos perinatais, principalmente das causas de morte, do aprimoramento da especificação das causas básicas após investigação e da codificação das causas de morte como parte do esforço de se reduzir a SC.

\section{REFERÊNCIAS}

1. Brasil. Ministério da Saúde. Secretaria de Vigilância em Saúde. Guia de vigilância em saúde. Brasília; 2014 [citado em 2017 ago. 15]. Disponível em: http://portalsaude.saude.gov.br/images/pdf/2014/novembro/27/guiavigilancia-saude-linkado-27-11-14.pdf.

2. Brasil. Ministério da Saúde. Transmissão vertical do HIV e sífilis: estratégias para redução e eliminação. Brasília; 2014 [citado em 2017 ago. 15]. Disponível em: http://www.aids.gov.br/sites/default/files/anexos/publicacao/2014/56610/ folder_transmissao_vertical_hiv_sifilis_web_pd_60085.pdf.

3. Domingues RMSM, Leal MC. Incidência de sífilis congênita e fatores associados à transmissão vertical da sífilis: dados do estudo Nascer no Brasil. Cad Saude Publica. 2016;36(6)

4. World Health Organization. Methods for surveillance and monitoring of congenital syphilis e elimination within existing systems. Geneva: WHO; 2011 [citado em 2017 ago. 15]. Disponível em: http://apps.who.int/iris/ bitstream/10665/44790/1/9789241503020_eng.pdf.

5. Brasil. Ministério da Saúde. Secretaria de Vigilância em Saúde. Secretaria de Atenção à Saúde. Manual de vigilância do óbito infantil e fetal e do Comitê de Prevenção do Óbito Infantil e Fetal. Brasília; 2009 [citado em 2017 ago. 15]. Disponível em: http://bvsms.saude.gov.br/bvs/publicacoes/ manual_obito_infantil_fetal_2ed.pdf.

6. Brasil. Ministério da Saúde. Agência Nacional de Vigilância Sanitária. Portaria 399, de 22 fevereiro 2006. Divulga o Pacto pela Saúde 2006. Consolidação do SUS e aprova as Diretrizes Operacionais do Referido Pacto. Diário Oficial da União [Internet], Brasília, 23 de fevereiro 2006 [citado em 2017 ago. 15]. Seção 1. Disponível em: https://www.nescon. medicina.ufmg.br/biblioteca/imagem/1831.pdf.

7. Organização Pan- Americana de Saúde. Resolução nº. CD50.R12, de 30 de setembro de 2010. Estratégia e plano de ação para a Eliminação da Transmissão materno-infantil do HIV e da sífilis congênita [Internet]. Washington, D.C.; 30 de mar 2010 [citado em 2017 ago. 15]. 62 seção. Disponível em: http://iris.paho.org/xmlui/bitstream/handle/123456789/414/ CD50.R12p.pdf? sequence $=4$ \&isAllowed $=y$.

8. Organização Pan- Americana de Saúde. Resolução nº. CD49.R19, de 02 de outubro de 2009. Eliminação de doenças negligenciadas e outras infecções relacionadas à pobreza. Washington, D.C.; 02 de out 2009 [citado em 2017 ago. 15]. 61 seção. Disponível em: http://new.paho.org/ hq/dmdocuments/2009/CD49.R19\%20(Eng.).pdf.

9. Brasil. Ministério da Saúde. Secretaria de Vigilância em Saúde. Boletim Epidemiológico 2015. Brasília; 2015 [citado em 2017 ago. 15]. Disponível em: http://www.aids.gov.br/sites/default/files/anexos/publicacao/2015/57978/_p_ boletim_sifilis_2015_fechado_pdf_p_18327.pdf.

10. Lorenzi DRS, Madi JM. Sífilis congênita como indicador de assistência pré-natal. Rev Bras Ginecol Obstet. 2001;23(10):647-52. http://dx.doi. org/10.1590/S0100-72032001001000006.

11. Domingues RMSM, Saraceni V, Hartz AMZ, Leal MC. Sífilis congênita: evento sentinela da qualidade da assistência pré-natal. Rev Saude Publica. 2013;47(1):14757. PMid:23703141. http://dx.doi.org/10.1590/S0034-89102013000100019.

12. Lima MG, Santos RFR, Barbosa GJ, Ribeiro GS. Incidência e fatores de risco para sífilis congênita em Belo Horizonte, Minas Gerais, 2001-2008. Ciênc Saúde Coletiva. 2011;18(2):499-506.

13. Brasil. Secretaria de Vigilância em Saúde. Indicadores e dados básicos da sífilis nos municípios brasileiros. Brasília [citado em 2017 ago. 15]. Disponível em: http://indicadoressifilis.aids.gov.br/.

14 Bowen V, Su J, Torrone E, Kidd S, Weinstock H. Increase in incidence of congenital Syphilis: United States, 2012-2014. MMWR Morb Mortal Wkly Rep. 2015;64(44):1241-1245.

15. Flores RLR. Sífilis congênita no município de Belém (Pará): análise dos dados registrados nos sistemas de informação em saúde (SINAN, SIM e SINASC) [dissertação]. Rio de Janeiro: FIOCRUZ; 2011.

16. Soeiro CMO, Miranda AE, Saraceni V, Santos MCS, Talhari S, Ferreira LCL. Sífilis na gestação e sífilis congênita no Estado do Amazonas, Brasil: uma avaliação utilizando ligação de bancos. Cad Saude Publica. 2014;30(4):71523. PMid:24896047. http://dx.doi.org/10.1590/0102-311X00156312.

17. Centro Brasileiro de Classificação de Doenças. Classificação Estatística Internacional de Doenças e Problemas Relacionados à Saúde - CID 10. [citado em 2017 ago. 15]. Disponível em: http://www.datasus.gov.br/cid10/ V2008/cid10.htm. 
18. Romero DE, Cunha CB. Avaliação da qualidade das variáveis sócio-econômicas e demográficas dos óbitos de crianças menores de um ano registrados no Sistema de Informações sobre Mortalidade do Brasil (1996/2001). Cad Saude Publica. 2006;22(3):673-84. PMid:16583111. http://dx.doi.org/10.1590/ S0102-311X2006000300022.

19. Barbuscia DM, Rodrigues-Junior AL. Completude da informação nas Declarações de Nascido Vivo e nas Declarações de Óbito, neonatal precoce e fetal, da região de Ribeirão Preto, São Paulo, Brasil, 2000-2007. Cad Saude Publica. 2011;27(6):1192-200. PMid:21710016. http://dx.doi.org/10.1590/ S0102-311X2011000600016.

20. Helena ETS, Rosa MB. Avaliação da qualidade das informações relativas aos óbitos em menores de um ano em Blumenau,1998. Rev Bras Saúde Matern Infant. 2003;3(1):75-83.

21. Frias PG, Szwarcwald CL, Morais No OL, Leal MC, Cortez-Escalante JJ, Souza Jr PRB, et al. Utilização das informações vitais para a estimação de indicadores de mortalidade no Brasil: da busca ativa de eventos ao desenvolvimento de métodos. Cad Saúde Pública. 2017 [citado em 2017 ago. 15];33(3):e00206015. Disponível em: http://www.scielo.br/scielo. php?script=sci_arttext\&pid=S0102-311X2017000305014\&lng=pt.

22. Saraceni V, Guimarães MHFS, Theme Fa MMT, Leal MC. Mortalidade perinatal por sífilis congênita: indicador da qualidade da atenção à mulher e à criança. Cad Saude Publica. 2005;21(4):1244-50. PMid:16021262. http:// dx.doi.org/10.1590/S0102-311X2005000400027.

23. Nascimento MI, Cunha AA, Guimarães EV, Alvarez FS, Oliveira SRSM, Bôas ELV. Gestações complicadas por sífilis materna e óbito fetal. Rev Bras Ginecol Obstet. 2012;34(2):56-62. PMid:22437763.
24. Brasil. Ministério da Saúde. Secretaria de Vigilância em Saúde. Manual de Instruções para o preenchimento da Declaração de Óbito. Brasília; 2011 [citado em 2017 ago. 15]. Disponível em: http://www.svs.aids.gov. br/download/manuais/Manual_Instr_Preench_DO_2011_jan.pdf.

25. Santo AH. Potencial epidemiológico da utilização das causas múltiplas de morte por meio de suas menções nas declarações de óbito, Brasil, 2003. Rev Panam Salud Publica. 2007;22(3):178-86. PMid:18062852. http://dx.doi. org/10.1590/S1020-49892007000800004.

26. Soares JAS, Horta FMB, Caldeira AP. Avaliação da qualidade das informações em declarações de óbitos infantis. Rev Bras Saúde Matern Infant. 2007;7(3):289-95.

27. Santos HG, Andrade SM, Silva AMR, Carvalho WO, Mesas AE, González AD. Concordância sobre causas básicas de morte infantil entre registros originais e após investigação: análise de dois biênios nos anos 2000 Rev Bras Epidemiol. 2014;17(2):313-22. PMid:24918406. http://dx.doi. org/10.1590/1809-4503201400020003ENG.

28. Santos SPC, Lansky S, Ishitani LH, França EB. Óbitos infantis evitáveis em Belo Horizonte: análise de concordância da causa básica, 2010-2011. Rev Bras Saúde Matern Infant. 2015;15(4):389-99.

29. Barbeiro FMS, Fonseca SC, Tauffer MG, Ferreira MS, Silva FP, Ventura PM, et al. Óbitos fetais no Brasil: revisão sistemática. Rev Saude Publica. $2015 ; 49(22)$

Recebido em: Ago. 05, 2017 Aprovado em: Set. 19, 2017 